\title{
DESARROLLO A ESCALA HUMANA DE LOS INMIGRANTES VENEZOLANOS EN SAN JOSÉ DE CÚCUTA*
}

\author{
https://doi.org/10.22395/seec.v22n51a2 \\ Eder Alexander Botello Sánchez ${ }^{* *}$ \\ Ángel David Ramírez Romero*** \\ Jorge Andrés Flores Rolón ${ }^{* * *}$
}

Recibido: 16 de febrero de 2019 - Aprobado: 04 de abril de 2019

\section{RESUMEN}

El presente estudio determina las diferencias entre las condiciones de vida de los inmigrantes venezolanos de la ciudad fronteriza de Cúcuta, Colombia, y su visión de desarrollo desde el enfoque del desarrollo a escala humana (DEH). Se usa una metodología cualitativa, con entrevistas focales, que permitieron la construcción de la matriz de necesidades axiológicas y existenciales con el software Atlas Ti, se elaboraron las redes semánticas de su condición actual y visión de desarrollo. Los resultados sugieren que: i) existen pobrezas en cada una de las necesidades estudiadas, ii) la visión del desarrollo está determinada en mayor medida por la estabilidad económica, iii) no existe un DEH en la sociedad de acogida, truncando la posibilidad de mejorar su calidad de vida.

\section{PALABRAS CLAVES}

Desarrollo; migración; inmigrantes; Venezuela; desarrollo a escala humana.

\section{CLASIFICACIÓN JEL}

O15, 131

\section{CONTENIDO}

Introducción; 1. Marco teórico; 2. Metodología; 3. Resultados; 4. Conclusiones; Bibliografía.

Este artículo de investigación realizado durante todo el periodo académico 2018, es fruto del trabajo de grado de los entonces estudiantes del programa de Economía de la Universidad de Pamplona: Ángel Ramírez y Andrés Flores. Al ser evidente el fenómeno migratorio en la frontera Colombo-venezolana de Cúcuta, Norte de Santander, se propuso indagar bajo la tutoría del profesor Alexander Botello, el desarrollo a escala humana y la migración. La información se levantó a partir del trabajo de campo realizado para la asignatura de Desarrollo Endógeno.

* Economista, Universidad de Pamplona, Villa del Rosario, Colombia. Especialista en Gerencia Financiera Universidad Libre, Cúcuta, Colombia. Magíster en Estudios y Gestión del Desarrollo, Universidad de la Salle, Bogotá, Colombia. Profesor e investigador del grupo de Investigación Economía y Estudios Fronterizos (Ecoesfront) de la Universidad de Pamplona, Villa del Rosario, Colombia. Correo electrónico: alexander. botello@unipamplona.edu.co

*** Economista, Universidad de Pamplona, Villa del Rosario, Colombia. Estudiante de inglés en intercambio cultural americano. Correo electrónico: angelramirez9605@gmail.com

..** Economista, Universidad de Pamplona, Villa del Rosario, Colombia. Estudiante de maestría en Filosofía en la Universidad de Salamanca, España. Correo electrónico: jorgeaflorezr@gmail.com 


\title{
HUMAN SCALE DEVELOPMENT OF VENEZUELAN IMMIGRANTS IN SAN JOSÉ DE CÚCUTA
}

\begin{abstract}
The present study determines the differences between living conditions of Venezuelan immigrants in the border city of Cúcuta, Colombia, and their comprehension and vision of development from the approach of the human scale development. With focal interviews, a qualitative methodology was applied, which allowed the construction of an axiological and existential needs matrix with the Atlas Ti software, semantic networks of their current condition and development vision were also developed. The results suggest that: i) poverty exists in each of the needs studied; ii) the idea of development is determined to a greater extent, by economic stability; iii) there is no human scale development in the host society, which cuts off the possibility of improving quality of life.
\end{abstract}

\section{KEYWORDS}

Development; migration; immigrants; Venezuela; Human scale development

\section{JEL CLASSIFICATION}

O15, I31

\section{CONTENTS}

Introduction; 1. Theoretical framework; 2. Methodology; 3. Results; 4. Conclusions; Bibliography.

\section{DESENVOLVIMENTO À ESCALA HUMANA DOS IMIGRANTES VENEZUELANOS EM SAN JOSÉ DE CÚCUTA}

\section{RESUMO}

Este estudo determina as diferenças entre as condições de vida dos imigrantes venezuelanos da cidade fronteiriça de Cúcuta, Colômbia, e sua visão de desenvolvimento sob a abordagem do desenvolvimento à escala humana (DEH). Utiliza-se uma metodologia qualitativa, com entrevistas focais que permitiram construir a matriz de necessidades axiológicas e existenciais com o software Atlas Ti; foram elaboradas as redes semânticas de sua condição atual e visão de desenvolvimento. Os resultados sugerem que: i) exista pobreza em cada uma das necessidades estudadas; ii) a visão do desenvolvimento esteja determinada em maior medida pela estabilidade econômica; iii) não exista um DEH na sociedade de acolhida, o que impede melhorar sua qualidade de vida.

\section{PALAVRAS-CHAVES}

Desenvolvimento; migração; imigrantes; Venezuela; desenvolvimento a escala humana.

\section{CLASSIFICAÇÃO JEL}

\author{
O15, I31
}

\section{CONTEÚDO}

Introdução; 1. Referencial teórico; 2. Metodologia; 3. Resultados; 4. Conclusões; Bibliografia. 


\section{INTRODUCCIÓN}

El estudio del desarrollo es diverso y versátil, y su paradigma ha venido cambiando en el marco de un mundo cada vez más globalizado y dinámico en el que el desplazamiento de personas de un lugar a otro, en busca de mejores condiciones de vida, ha tenido un lugar predominante en la historia de la humanidad. Estas migraciones son todas distintas, no solo en cuanto a magnitud e intensidad, sino también en cuanto a sus móviles y perspectivas.

En ese orden de ideas, el estudio del desarrollo en población migrante requiere de un enfoque teórico que sitúe al ser humano en el centro de la discusión. Un enfoque que no asuma exclusivamente el desarrollo como equivalente a una disponibilidad cada vez mayor de bienes y servicios, de niveles de renta elevados o de tasas crecientes del nivel de capital disponible, sino uno que considere los procesos micro del desarrollo, aquel que apueste por satisfacer las necesidades humanas fundamentales, lo que representa una visión del desarrollo que "obliga a ver y a evaluar el mundo, las personas y sus procesos, de una manera distinta a la convencional" (Max-Neef, Elizalde y Hopenhayn, 2010, p. 15).

De acuerdo con lo anterior, un enfoque que contemple al ser humano como elemento receptivo-gestor del desarrollo, permite incluir en el análisis de las variables las actividades humanas que inciden en su desarrollo. De esta forma trata de contextualizar la dinámica socioeconómica de un grupo social y ajustar la cuestión del desarrollo a sus requerimientos particulares. En este artículo el grupo social objeto de estudio es la población inmigrante que está de paso en la casa refugio Los Scalabrinis ${ }^{1}$ de la ciudad de San José de Cúcuta, Colombia.

En la región fronteriza entre Colombia y Venezuela se presenta una creciente migración de ciudadanos venezolanos hacia Colombia, el motivo de ello ha sido la crisis socioeconómica en Venezuela; de acuerdo con el balance preliminar presentado por el informe de la Comisión Económica para América Latina y el Caribe (Cepal), durante el 2017: "El PIB de la economía venezolana se redujo por cuarto año consecutivo, lo que supone una contracción acumulada del 31,9\% respecto del PIB de 2013. De igual forma, 2017 sería el cuarto año con tasas de inflación de más de dos dígitos y el segundo con tasas superiores al $300 \% "$ (Cepal, 2018).

La congregación religiosa Scalabrini, fue fundada en Italia en 1887 por Juan Bautista Scalabrini. Se creó con el objetivo de ayudar a los inmigrantes y refugiados políticos en la mayoría de países del mundo. En la ciudad de Cúcuta esta misión hace presencia desde el año 1978 en el barrio Sevilla y producto de la problemática migratoria venezolana, el año 2018 ha alcanzado niveles de población migrante desde Venezuela hacia Colombia en constante crecimiento. Esta misión de la Iglesia católica brinda hospedaje y alimento por un tiempo determinado, esto permitió recolectar la información con el mismo grupo de personas. 
En este contexto de espacio fronterizo y de crisis económica en Venezuela, hacia finales de 2017 "más de 550,000 ciudadanos venezolanos habían cruzado la frontera hacia Colombia, lo que representa un incremento cercano al $62 \%$ respecto al mismo informe presentado en 2016" (Migración Colombia, 2017). Ahora bien, en la ciudad de Cúcuta, la población inmigrante no consigue escapar del todo de la crisis que motivó su desplazamiento. De acuerdo con los datos del Departamento Administrativo Nacional de Estadística (DANE), un 22,4\% de los hogares en Cúcuta presentaba necesidades básicas insatisfechas (DANE, 2011). Para el caso de la pobreza monetaria y la pobreza monetaria extrema, el índice durante 2017 fue de 33,5 \% y 5,3\% respectivamente y, el coeficiente de GINI, fue de 0,426 (DANE, 2018a).

Adicionalmente, la tasa de desempleo para Cúcuta durante el trimestre octubre-diciembre de 2017, se ubicó en 14,0\%, superando el 13,8\% del mismo trimestre durante el 2016 (DANE, 2018b). Así las cosas, de acuerdo con los indicadores mencionados, la crisis se hace visible en ambos lados de la frontera y si bien los migrantes buscan una mejor calidad de vida en las sociedades de acogida, una proporción de la población local suele asumir una posición de rechazo, en donde se observan casos de xenofobia, esto dado que la migración es percibida en ocasiones en forma de amenaza o riesgo. Para el caso de los habitantes de Cúcuta, la creciente entrada de ciudadanos venezolanos, sumado a las condiciones sociales y económicas de desempleo e informalidad laboral persistentes en la ciudad, dificultan la relación con la población inmigrante, con lo cual surgen tensiones y conflictos sociales.

Frente a esta problemática, este artículo aporta a la comprensión del fenómeno de la inmigración desde el enfoque teórico del desarrollo a escala humana (DEH) de Manfred Max-Neef, lo cual permite captar una imagen más amplia de los inmigrantes venezolanos a su llegada a la ciudad de Cúcuta, a partir de sus necesidades axiológicas y existenciales. De esta manera, el enfoque DEH se emplea para determinar las diferencias que puedan existir entre las condiciones de vida de los inmigrantes venezolanos que residen en la casa de refugio Scalabrini y su visión del desarrollo. Para ello el documento se compone de cuatro secciones: la primera expone brevemente las escuelas del desarrollo, sus representantes y postulados teóricos; seguido del método de investigación cualitativo utilizado, en el que se hace uso del software Atlas Ti, para el procesamiento de la información obtenida en el trabajo de campo con la población objetivo; en la tercera sección se presentan los resultados principales y, finalmente, las conclusiones.

\section{MARCO TEÓRICO}

A partir de los años cincuenta del siglo XX, derivado del análisis histórico-económico del proceso de desarrollo de los países industrializados, se formularon modelos y 
estrategias de política económica que involucraron transformaciones estructurales y la correspondiente asignación al papel del gobierno en la planeación del desarrollo en los países no industrializados (Meier, 2002), en los años siguientes, producto de las diferencias conceptuales sobre el desarrollo, surgieron diversas escuelas teóricas en las que se inscribirían los economistas del desarrollo. La primera sección de este apartado expone de manera cronológica las escuelas del desarrollo, la sección media presenta la escuela alternativa del desarrollo y su enfoque multidisciplinar, posteriormente, la propuesta de desarrollo a escala humana (DEH), definida por Max-Neef, Elizalde y Hopenhayn (2010, p. 7) como "un aporte sustancial para la construcción de un nuevo paradigma del desarrollo, menos mecanicista y más humano".

\subsection{Escuelas del desarrollo}

De acuerdo con Hobsbawn (1999), la primera mitad del siglo XX no puede disociarse de las dos guerras mundiales o, la larga guerra de 31 años de 1914-1945, y las catástrofes políticas, económicas y sociales de entreguerras, como la Revolución rusa de 1917 y las alarmantes experiencias económicas que culminaron en la Gran Depresión de 1929-1933. La suma de estos eventos, en los años posteriores al fin de la Segunda Guerra Mundial, determinaron el surgimiento de nuevas interpretaciones de la realidad socioeconómica global, definida por Leal (2012, p. 214) como la de un mundo en el que al "conjunto de los países más antiguos y de industrialización temprana se le llamó Primer Mundo, mientras que, al grupo de aquellos atrasados, como los de América Latina, y los que se crearon con las guerras de descolonización se lo calificó de Tercer Mundo".

En el marco de esta nueva categorización de los países de acuerdo con su nivel de industrialización, Hidalgo-Capitán (2011) argumenta que a partir de los años cuarenta y cincuenta del siglo XX, diversos autores comenzaron a teorizar sobre el desarrollo de los países pobres. Las variaciones teóricas y conceptuales, propuestas por estos autores, determinaron el surgimiento de las escuelas del desarrollo, su concepción del desarrollo y el proceso por el cual se habrían de guiar los países del tercer mundo en la carrera por la superación de la pobreza.

La primera generación de teóricos del desarrollo optó, según Girola (2008, p. 14), por el "proceso de industrialización y de crecimiento del mercado interno". La escuela de la modernización, de acuerdo con Meier (2002, p. 3), representada por "las etapas del crecimiento de Rostow, el crecimiento equilibrado de Nurkse y el gran empuje de Rosenstein-Rodan", priorizaron la acumulación de capital, con ello, el desarrollo equivalía a crecimiento económico, entendido este como el aumento sostenido de la renta durante un largo periodo de tiempo (Mora, 2006). 
La economía mundial comprende dos grandes polos que interactúan: centro y periferia. Sus estructuras productivas son disímiles, pues mientras en la segunda son heterogéneas y especializadas, en la primera son homogéneas y diversificadas. Estas diferencias condicionan el tipo de intercambio comercial y de transferencias tecnológicas en la economía mundial. En el largo plazo, la evolución económica de este sistema conduce a un rezago productivo y tecnológico en la periferia y a un deterioro de sus términos de intercambio (Rosales, 1988).

Frente a la respuesta de las nuevas escuelas del desarrollo que promovían principalmente una industrialización de las economías a partir de la superación de la dependencia, la escuela neoliberal impuso la tesis de que el subdesarrollo estaba definido como una condición de desequilibrio económico en las naciones no industrializadas, producto de una excesiva regulación económica, que desestimulaba la libre circulación de bienes y capital, como elementos necesarios para dinamizar el libre mercado y promover el desarrollo.

Del renovado interés por integrar al análisis economicista del desarrollo, aparecen otras disciplinas sociales a partir de las cuales entender el fenómeno de subdesarrollo y superar los postulados neoclásicos del desarrollo representados por la escuela neoliberal, surge la escuela alternativa y su visión del desarrollo definida por Hidalgo-Capitán (2011, p. 302) como:

Una situación de privación de la satisfacción efectiva de las necesidades básicas de los individuos, debido a la escasez de provisión de bienes y servicios necesarios para satisfacer dichas necesidades o la falta de capacidad de los individuos para hacer un uso efectivo de ellos. Por tanto, la mejor manera de salir del subdesarrollo es aumentar las capacidades de los individuos para que puedan satisfacer de forma efectiva sus necesidades básicas.

\subsection{Escuela alternativa}

La concepción del desarrollo desde una perspectiva multidisciplinar ha contribuido a la evolución teórica respecto a cuál es el mejor camino para disminuir los niveles de pobreza. Sin embargo, las diferencias conceptuales a partir de las cuales se diseñan las estrategias del desarrollo han derivado en enfoques diversos, todos ellos enmarcados en la categoría de la escuela alternativa. Esta escuela pretende reivindicar al ser humano en la ciencia económica, es decir, se procura superar el carácter mecanicista y racional del sujeto económico propuesto en la teoría económica neoclásica. De acuerdo con Bustelo (1998, p. 143) "a mediados de los años sesenta se dio inicio a una nueva fase en la historia del pensamiento económico sobre desarrollo. A la importancia otorgada al crecimiento sucedió una preocupación por los objetivos propiamente dichos del desarrollo, esto es por los fines y no por los medios". 
De ahí que, en los diferentes enfoques de esta escuela, el objetivo sea una comprensión más efectiva de las realidades en las que se desarrollan las personas y los grupos sociales, a partir de esto, cada enfoque establece una variable de consideración, dando como resultado los enfoques presentados en la tabla 1.

Tabla 1. Centro de los enfoques del desarrollo en la escuela alternativa

\begin{tabular}{|c|c|c|c|c|c|}
\hline $\begin{array}{l}\text { Desarrollo } \\
\text { humano }\end{array}$ & $\begin{array}{l}\text { Desarrollo } \\
\text { territorial }\end{array}$ & $\begin{array}{l}\text { Desarrollo } \\
\text { sostenible }\end{array}$ & $\begin{array}{c}\text { Desarrollo con } \\
\text { perspectiva de } \\
\text { género }\end{array}$ & Posdesarrollo & $\begin{array}{l}\text { Altermundiza- } \\
\text { ción }\end{array}$ \\
\hline $\begin{array}{l}\text { La satisfac- } \\
\text { ción de las ne- } \\
\text { cesidades de } \\
\text { los individuos }\end{array}$ & $\begin{array}{l}\text { La dimen- } \\
\text { sión territorial } \\
\text { del desarrollo } \\
\text { con sus múl- } \\
\text { tiples varian- } \\
\text { tes (desarro- } \\
\text { llo regional, lo- } \\
\text { cal, urbano, ru- } \\
\text { ral, endógeno, } \\
\text { descentraliza- } \\
\text { do, de abajo- } \\
\text { arriba, etc.) }\end{array}$ & $\begin{array}{l}\text { La dimensión } \\
\text { ambiental del } \\
\text { desarrollo }\end{array}$ & $\begin{array}{l}\text { El papel de las } \\
\text { mujeres en el } \\
\text { desarrollo }\end{array}$ & $\begin{array}{l}\text { La negación } \\
\text { del estilo } \\
\text { de vida de } \\
\text { los países } \\
\text { desarrollados } \\
\text { como modelo } \\
\text { a seguir. }\end{array}$ & $\begin{array}{l}\text { El orden eco- } \\
\text { nómico inter- } \\
\text { nacional y en } \\
\text { la regulación } \\
\text { de la globali- } \\
\text { zación. }\end{array}$ \\
\hline
\end{tabular}

Fuente: elaboración propia a partir de Hidalgo-Capitán (2011).

\subsection{Desarrollo a escala humana (DEH)}

El aporte de Max-Neef a la escuela alternativa es peculiar, proporciona nuevos conceptos y críticas a la economía neoclásica, tanto a su objeto de estudio como en su teoría. De esta manera, plantea una nueva propuesta para pensar el desarrollo, con un enfoque pluralista desde su estudio multidisciplinario, hasta las diferencias cualitativas de los individuos estudiados. En palabras de Max-Neef, Elizalde y Hopenhayn (2010, p. 11), "ello implica, por cierto, asumir como principio algo que pareciera olvidarse con demasiada frecuencia: que la economía está para servir a las personas, y no las personas para servir a la economía".

Para la época en que Sen ya era reconocido por su aporte al desarrollo desde la escuela alternativa, en América Latina, hacia mediados de los años 80, Manfred Max-Neef desarrolla su obra más sobresaliente, Desarrollo a Escala Humana, publicada en 1993. El contexto sobre el cual surgen estas ideas está marcado por los diferentes modelos que afrontó América Latina durante las cuatro décadas después de la Segunda Guerra Mundial. Como lo expone en su libro, las escuelas tradicionales fueron promotoras de ideas y recetas para ponerse a prueba en los países subdesarrollados. Solo un enfoque multidisciplinario permite comprender las nuevas 
calamidades sociales y problemas complejos que el reduccionismo no ha permitido resolver satisfactoriamente. "Si las políticas económicas afectan a la totalidad de la sociedad, los economistas ya no pueden pretender que su única preocupación sean los problemas económicos" (Max-Neef et al., 2010, p. 39).

De manera analógica a la intriga por la razón de las hambrunas y los problemas referentes a la pobreza en el mundo, Max-Neef aborda las cuestiones respecto a las medidas adoptadas por los países latinoamericanos, las cuales a manera de péndulo entre el desarrollismo y el liberalismo crean ciclos económicos, en donde la población menos favorecida queda al margen tanto de la representación política como de la participación económica. En palabras de Max-Neef et al. (2010, p. 47) este es el desafío del futuro:

Reconocer y evaluar las patologías colectivas que los diversos sistemas sociopolíticos son capaces de provocar -cada uno a su manera y con su propia intensidadcomo resultado del bloqueo sistemático de necesidades tales como entendimiento, protección, identidad, afecto, creatividad y libertad... Los economistas, especialmente los ubicados en posiciones de influencia, deberían hacer su propio esfuerzo de honesta autocrítica para descubrirlos y reconocerlos.

\section{METODOLOGÍA}

El presente artículo hace uso del método de investigación cualitativo, como señala Hernández Sampieri, Fernández y Bautista (2014, p.11) "Gusca describir, comprender e interpretar los fenómenos, a través de las percepciones y significados producidos por las experiencias de los participantes". Ahora bien, este tipo de investigación "se selecciona cuando el propósito es examinar la forma en que los individuos perciben y experimentan los fenómenos que los rodean, profundizando en sus puntos de vista, interpretaciones y significados" (Hernández Sampieri et al., 2014, p. 358).

Max-Neef establece que el desarrollo se debe analizar desde un enfoque antropocéntrico, lo que se pretende es reconstruir la realidad a partir de las interpretaciones subjetivas de los individuos, por esta razón, la investigación cualitativa es la que mejor se ajusta al objetivo del DEH, en donde el desarrollo debe procurar satisfacer las necesidades humanas fundamentales, todo ello basado en la lectura que los sujetos hacen de su realidad.

El alcance de la investigación es descriptivo², dado que supone la delimitación de la situación por la que pasan los migrantes según sus propios argumentos,

\footnotetext{
2 Para Veiga de Cabo, de la Fuente y Zimmermann "la investigación de tipo descriptivo se limita a medir la presencia, característica o distribución de un fenómeno en una población" (2008, p. 82), para el caso de la presente investigación, la dimensión del desarrollo de una población en un momento determinado del tiempo.
} 
percepciones y expectativas. Para ello la investigación se divide en dos etapas metodológicas: la primera trata de la construcción de la matriz axiológica, según Max-Neef et al. (2010, p. 41):

Las necesidades humanas pueden desagregarse conforme a múltiples criterios, (...) según categorías existenciales y según categorías axiológicas. Esta combinación permite operar con una clasificación que incluye, por una parte, las necesidades de ser, tener, hacer y estar y, por la otra, las necesidades de subsistencia, protección, afecto, entendimiento, participación, ocio, creación, identidad y libertad. Ambas categorías de necesidades pueden combinarse con la ayuda de una matriz.

La construcción de la matriz tiene en cuenta dos postulados de Max-Neef. Primero, las necesidades fundamentales son finitas, pocas y clasificables, segundo, las necesidades humanas son las mismas en toda cultura y tiempo, lo que cambian son los satisfactores.

Finalmente, la segunda etapa constituye la descripción del imaginario social ${ }^{3}$ en torno al desarrollo a escala humana por parte de la población objeto de estudio. Para ello se llevó a cabo el proceso de codificación planteado por Hernández Sampieri et al. (2014), el cual tiene dos planos o niveles: en el primero, se codifican las unidades en categorías; en el segundo, se comparan las categorías entre sí para agruparlas en temas y buscar posibles vinculaciones. La codificación de la información se hace a través de la herramienta especializada en este tipo de investigaciones: el software Atlas Ti, el cual facilita la segmentación de datos cualitativos de acuerdo con las categorías establecidas por el investigador, lo que permite generar conteos y relaciones entre categorías, temas o documentos primarios.

La técnica de recolección de información incluye la elección de una muestra conceptual $^{4}$ en la casa refugio Los Scalabrini, ubicada en la ciudad de Cúcuta. La información provino de 25 casos (cinco grupos conformados cada uno por cinco personas), a los que se les realizó una entrevista focal y en donde la ejecución de la actividad se gestó en cuatro etapas: i) romper el hielo con una actividad lúdica; ii) presentación de las propuestas del investigador y reglas de la actividad; iii) conformación de los grupos y aplicación del instrumento semiestructurado con los aportes de la población objeto de estudio y iv) compartir, y agradecimiento a las personas participantes.

3 Para Agudelo, el concepto de imaginario comprende "un conjunto real y complejo de imágenes (de lo que somos y queremos) que aparecen para provocar sentidos diversos, sentidos que acaecen, se instituyen y abren mundo" (2011, p. 1).

4 Según Hernández Sampieri et al. (2014), esta muestra se escoge en base a la teoría o concepto que sirvan para el fin de la investigación. 


\section{RESULTADOS}

Max-Neef argumenta que se necesita una teoría de las necesidades para el desarrollo, este enfoque propone la aplicación de tres tipos de matrices: una matriz positiva, negativa y una utópica, conociendo de antemano que la elaboración de la matriz no está exenta de arbitrariedades. Este artículo construye, a partir de las entrevistas de la población inmigrante venezolana en la casa refugio Los Scalabrini en la ciudad de San José de Cúcuta para el 2018, la matriz negativa y dos redes semánticas; la primera expone la percepción de calidad de vida y la segunda, presenta las condiciones ideales que configuran una buena calidad de vida para los inmigrantes.

De acuerdo con la tabla 2, se tiene que la matriz negativa clasifica los satisfactores, destructores o violadores que suplen las necesidades existenciales en relación con las necesidades axiológicas. Los satisfactores que forman parte del ser, son de carácter destructivo como el miedo, la discriminación, la desconfianza e inestabilidad, entre otros, debido a que dificultan la satisfacción actual de necesidades como el afecto, la protección, la subsistencia y la identidad. Estas formas de satisfacer la necesidad del ser se encuentran muy relacionadas con la integración a la sociedad de acogida, la cual usualmente percibe la inmigración como una invasión que depreda las oportunidades del entorno, esta percepción se aprecia en lo expuesto por Jiménez y García (2013, p. 36): "La inmigración aparece como una pirámide donde siempre hay alguien por debajo o por encima. Hacer resurgir cada cierto tiempo el temor al 'extranjero que viene a sustraernos lo nuestro' es un método bastante eficaz en un periodo de crisis en que el desempleo amenaza".

Continuando con la necesidad del ser, la forma de suplir la participación se ve afectada por la fragmentación de los vínculos sociales y familiares de los inmigrantes, debido a que no hay cohesión social entre estos, la situación se podría explicar, en parte, porque los inmigrantes en su mayoría son hombres en un $56 \%$ como se demostró en el Informe sobre la movilidad humana venezolana presentado por Migración Colombia (2018). "En promedio, cada hogar que se está asentando en Colombia está compuesto por 1,7 personas, lo que indica que la mayoría de quienes han llegado vienen solos. Eso no solo habla de la calamidad que representa esa fracturación de las familias" (Migración Colombia, 2018).

Por otra parte, desde el ser, la libertad se satisface de manera negativa con el estado de soledad, siendo un satisfactor destructor, toda vez que la cultura de los inmigrantes tiene como pilar del desarrollo, el entorno familiar, complementariamente, la imagen negativa de sus compatriotas (creencia de la población autóctona, acerca del aumento de la delincuencia por la llegada de venezolanos) en la ciudad genera una desconfianza para asociarse entre ellos. 
Tabla 2. Matriz negativa de la población objeto de estudio

\begin{tabular}{|c|c|c|c|c|}
\hline & Ser & Tener & Hacer & Estar \\
\hline $\begin{array}{l}\text { Subsisten- } \\
\text { cia }\end{array}$ & $\begin{array}{l}\text { Inestabilidad } \\
\text { Individualismo }\end{array}$ & $\begin{array}{l}\text { Falta de oportunidades } \\
\text { Falta de regularidad } \\
\text { Falta de representati- } \\
\text { vidad }\end{array}$ & $\begin{array}{l}\text { Necesidad } \\
\text { de trabajar } \\
\text { Pelear } \\
\text { Tratar de ser } \\
\text { escuchados }\end{array}$ & $\begin{array}{l}\text { Ambiente hostil } \\
\text { Espacio de desconfianza }\end{array}$ \\
\hline Protección & $\begin{array}{l}\text { Discriminación } \\
\text { Inseguridad } \\
\text { Opresión } \\
\text { Menosprecio } \\
\text { Miedo }\end{array}$ & $\begin{array}{l}\text { Falta de trabajo y dinero } \\
\text { Falta de legalidad y ac- } \\
\text { ceso a servicios de salud }\end{array}$ & $\begin{array}{l}\text { Desconfiar } \\
\text { Rebuscarse } \\
\text { la manera de } \\
\text { ganar algo } \\
\text { de dinero } \\
\text { Estar a la } \\
\text { defensiva }\end{array}$ & $\begin{array}{l}\text { Territorio sin legislación in- } \\
\text { clusiva }\end{array}$ \\
\hline Afecto & $\begin{array}{l}\text { Desconfianza } \\
\text { Individualismo } \\
\text { Prejuicio } \\
\text { Indiferencia }\end{array}$ & $\begin{array}{l}\text { Gente maleducada } \\
\text { Falta de familiares y } \\
\text { amigos }\end{array}$ & $\begin{array}{l}\text { Discriminar } \\
\text { Juzgar } \\
\text { Reprimir }\end{array}$ & $\begin{array}{l}\text { Espacios de desconfianza } \\
\text { Sociedad desconocida } \\
\text { Espacios no aptos para de- } \\
\text { mostrar afecto }\end{array}$ \\
\hline $\begin{array}{l}\text { Entendi- } \\
\text { miento }\end{array}$ & $\begin{array}{l}\text { Desaliento } \\
\text { Depresión }\end{array}$ & $\begin{array}{l}\text { Conocimientos no reco- } \\
\text { nocidos }\end{array}$ & $\begin{array}{l}\text { Resignar } \\
\text { Aplazar }\end{array}$ & $\begin{array}{l}\text { Sistema educativo de la ciu- } \\
\text { dad inflexible }\end{array}$ \\
\hline $\begin{array}{l}\text { Participa- } \\
\text { ción }\end{array}$ & $\begin{array}{l}\text { Fragmentación } \\
\text { Marginación }\end{array}$ & $\begin{array}{l}\text { Exclusión } \\
\text { Falta de participación } \\
\text { política }\end{array}$ & $\begin{array}{l}\text { Excluir } \\
\text { buscar el } \\
\text { beneficio } \\
\text { propio }\end{array}$ & $\begin{array}{l}\text { Entes territoriales } \\
\text { Falta de asociaciones de } \\
\text { refugiados e inmigrantes } \\
\text { Aislamiento }\end{array}$ \\
\hline Ocio & $\begin{array}{l}\text { Estrés } \\
\text { Impaciencia }\end{array}$ & $\begin{array}{l}\text { Falta de recursos } \\
\text { Exclusión social }\end{array}$ & $\begin{array}{l}\text { Cohibir } \\
\text { Coartar }\end{array}$ & $\begin{array}{l}\text { Falta } \\
\text { Lugares de esparcimiento } \\
\text { incluyentes }\end{array}$ \\
\hline Creación & $\begin{array}{l}\text { Falta de } \\
\text { recursos }\end{array}$ & $\begin{array}{l}\text { Difícil acceso al crédito } \\
\text { Escasez de oportuni- } \\
\text { dades }\end{array}$ & Sobrevivir & $\begin{array}{l}\text { Falta de talleres de educa- } \\
\text { ción financiera }\end{array}$ \\
\hline Identidad & $\begin{array}{l}\text { Prejuicios } \\
\text { Bajo autoes- } \\
\text { tima } \\
\text { Miedo }\end{array}$ & $\begin{array}{l}\text { Falta de incentivos para } \\
\text { ser como se es }\end{array}$ & Ocultar & $\begin{array}{l}\text { Falta de entornos para en- } \\
\text { cuentros que resalten hábi- } \\
\text { tos y costumbres }\end{array}$ \\
\hline Libertad & $\begin{array}{l}\text { Miedo } \\
\text { Soledad } \\
\text { Rivalidad }\end{array}$ & $\begin{array}{l}\text { No hay derecho al tra- } \\
\text { bajo } \\
\text { No hay respeto por el } \\
\text { derecho a la igualdad } \\
\text { No hay confianza en las } \\
\text { autoridades } \\
\text { Confianza en la Iglesia y } \\
\text { personas cercanas }\end{array}$ & $\begin{array}{l}\text { Cohibir } \\
\text { Callar }\end{array}$ & $\begin{array}{l}\text { Falta de espacios que ga- } \\
\text { ranticen la igualdad y la } \\
\text { seguridad } \\
\text { Iglesia }\end{array}$ \\
\hline
\end{tabular}

Fuente: elaboración propia. 
Ortiz, Manzanera y Clavero, (2013) citan a Prieto-Lacaci (1993), el cual se refiere a los migrantes latinoamericanos como una población con débil cultura sobre el asociacionismo, como también menciona que la mujer tiene un efecto notorio en estos temas. El hecho de que ellas sean más unidas se debe a la tarea del cuidado de los niños y su cooperativismo con las demás para llevar a cabo estas actividades mientras los hombres salen en busca del sustento diario, como lo mencionan Ortiz et al. (2013), las mujeres tienen un mayor compromiso de participación que los hombres en asociaciones de carácter social y organizaciones de ayuda al inmigrante, mientras que los hombres participan más en asociaciones como clubes deportivos, grupos educativos y culturales.

"Acá no nos sentimos libres, primero porque no conozco, no tengo a nadie acá". Participante número 7 , grupo focal 4.

Otro satisfactor destructor en la necesidad del ser es la discriminación, tanto en hombres como en mujeres, la cual se experimenta en los sitios públicos donde pueden satisfacer la necesidad de ocio, como lo expresó un participante del grupo focal:

"Uno va a salir a jugar algún deporte y lo miran mal, ¿ccómo uno hace ahí?" Participante número 15 , grupo focal 3.

Para el caso de la necesidad del tener, se tienen satisfactores negativos como la legislación laboral excluyente, el adverso entorno cívico al que llegan, la difícil estancia regular y la falta de igualdad, y también bienes económicos ausentes como el dinero, el crédito y autoridades confiables. Lo anterior ha afectado de manera general su integración social dado que los satisfactores negativos mencionados representan una exclusión que se traduce finalmente en discriminación, como se ha visto en los demás fenómenos migratorios que se han dado en el mundo, Ortiz et al. (2013, p. 141) argumentan que:

La población inmigrante comparte los problemas de la sociedad en la que se integra sin obviar por supuesto que se convierte en uno de los colectivos más vulnerables a la exclusión social al no disfrutar de los derechos sociales que en cualquier sociedad configuran la integración social: el trabajo, el pleno ejercicio de la ciudadanía y el derecho a la vivienda.

Un bien material de la necesidad del tener, que más adelante tomará importancia en el análisis de las redes semánticas, es la vivienda, la cual está condicionada en mayor medida por un mercado laboral con escasas oportunidades que dificulta 
el acceso a dicho bien, lo que conlleva a que los inmigrantes acudan al refugio y en última instancia a las calles de la ciudad para satisfacer necesidades como la protección y el afecto. Como citaron Hernández y López (2013, p.170) a Hernández (2010) "en suma, los problemas relacionados con la esfera residencial son uno de los principales desencadenantes de procesos de exclusión social." Los obstáculos de esa integración eventualmente hacen que los inmigrantes queden privados de derechos de ciudadanía, esto conlleva a acciones excluyentes y de nuevo discriminantes pero que actúan en diferentes aspectos como el empleo, la vivienda, la participación y la sanidad.

Ahora bien, al analizar la necesidad del hacer, se destacan acciones como el tratar de ser escuchados, sobrevivir, en ocasiones callar y ocultar, las cuales se desprenden de la unión de las necesidades anteriores, también se tiene que se ha gestado prejuicio por parte de los inmigrantes hacia la población de acogida. Lo anterior es producto de una nula integración social, la necesidad de esta se ve reflejada por la presencia de xenofobia y exclusión cuya mezcla impide la inclusión social mediante la educación y el trabajo, lo cual podría generar un problema público como el incremento de cinturones de miseria, el pandillismo y el aumento de la inseguridad como se ha visto en experiencias de otros países.

En este orden de ideas, para la necesidad de estar, los obstáculos expuestos a la inclusión social afectan de manera negativa las necesidades de libertad e identidad, las cuales se ven relacionadas con el entorno, como se observa en la tabla 2, los satisfactores destructores como lo son el prejuicio, el miedo y la baja autoestima son aspectos que generan una inestabilidad emocional en los inmigrantes y en el peor de los casos influyen en su identidad y la manera de percibir el entorno como desigual y hostil.

Esto mismo ocurrió en el continente europeo con la llegada de inmigrantes de diferentes países con lenguas y costumbres distintas lo que generó conflictos de identidad en los inmigrantes, al pertenecer a una cultura por herencia familiar y nacer en otra, esto conlleva a la ambigüedad extranjeros/pero no extranjeros. Jiménez y García (2013) argumentan que la aculturación va a provocar conflictos importantes que pueden incidir en el proceso de construcción de su identidad, sin embargo, en el caso colombovenezolano se vive un contexto distinto. Según Zambrano, Araque y Ravelo (2014, p. 5):

La frontera Norte de Santander -Estado Táchira, es una línea imaginaria que no se encuentra contemplada por las personas que diariamente atraviesan los puentes para "cambiar de país"; es algo tan habitual que le restan importancia, siendo un 
hecho apenas evidente ya que, al pasar del otro lado de la franja, se encuentran con el mismo idioma y culturas similares.

No obstante, al ver que la mayoría de los inmigrantes provienen de Estados del interior de Venezuela donde no se puede maniobrar la crisis con el paso de la frontera para abastecerse, los inmigrantes ven la diferencia en muchos aspectos de la cultura colombiana, la cual les genera incertidumbre.

"Porque ya uno sabe cómo es el venezolano allá, mientras que aquí uno no sabe cómo son las personas". Participante número 2 grupo focal 2.

En ello, los inmigrantes perciben los problemas de la ciudad, al no encontrar la forma de suplir necesidades de empleo, oportunidades, seguridad social y regularidad (documentación). Estos aspectos son fundamentales en relación con la pobreza y la participación en el mercado. Finalmente, la necesidad de estar se ve insatisfecha debido a que la ciudad no ofrece muchas alternativas para que esta población prospere. Lo anterior se observa en la tabla 1, al resaltar la falta de legislación inclusiva, de lugares de esparcimiento integradores y promotores para la satisfacción de necesidades como el entendimiento, este último, de vital importancia en una integración económica que podría dar paso una movilidad social para alcanzar dos pilares que provocan el movimiento migratorio: la estabilidad económica y emocional.

Al abordar de manera global la matriz negativa de la tabla 2, se pueden denotar a partir del enfoque de Max-Neef, pobrezas en cada una de las combinaciones entre las necesidades existenciales y axiológicas; no obstante, Max-Neef argumenta que esas pobrezas son también potencialidades, de manera que se resalta desde otra perspectiva la voluntad por parte de la población inmigrante de superar retos, estudiar y conocer la forma de generar ingresos, como también de ser escuchados, de tener voz en las decisiones políticas y hacer su aporte desde la experiencia. Así, la conformación de esta matriz la hace propositiva y crítica, debido a que los satisfactores que la componen describen sus condiciones de vida en la ciudad de acogida, como también los distintos aspectos en los que se puede mejorar la integración de su estancia en la ciudad.

A pesar de las adversidades expuestas en párrafos anteriores, en la ciudad de Cúcuta y más específicamente en la casa refugio Los Scalabrini, los inmigrantes encuentran condiciones o situaciones positivas en medio de la coyuntura, sin embargo, estos satisfactores no representan un pleno desarrollo a escala humana para la población de estudio. Una descripción del fenómeno migratorio y que encierra 
lo hasta ahora descrito en torno a las condiciones sociales de los inmigrantes venezolanos es lo señalado por Bañón y Molina (2013, p. 15): "Hay millones de personas que no tienen nada que perder y a los que no les importa lo desesperado de las condiciones en las que tengan que emigrar. De hecho, muchas veces la inmigración aparece como la única esperanza de poder sobrevivir con mayor dignidad: ellos o sus familias."

\subsection{Percepción acerca de la calidad de vida de la población inmigrante venezolana de la casa refugio Los Scalabrini en la ciudad de Cúcuta}

En las siguientes redes es posible identificar proximidades semánticas (acercamiento entre categorías) que surgen a partir de los vínculos que existen entre las categorías al interior de cada discurso. Ahora bien, estas proximidades no tienen una forma estandarizada para el análisis e interpretación, por lo que permite una flexibilidad sin comprometer la rigurosidad del método (esta es una de las ventajas del método cualitativo).

\subsubsection{Red semántica percepción calidad de vida}

Las medidas que se han llevado a cabo en los países europeos como Francia y Alemania han demostrado el avance integrador de las políticas migratorias, ante la usual perspectiva de amenaza que las sociedades de acogida ven en el fenómeno migratorio, esto se relaciona con la integración social y económica que busca acabar con la exclusión social que se da en las sociedades receptoras, como lo mencionan Subirats et al. (2004, p. 32) "La Unión Europea adoptó este término para enmarcar sus iniciativas y preocupaciones en materia social, y se viene utilizando el concepto de exclusión social como expresión de la ruptura de personas o colectivos con los lazos o vínculos sociales que les confieren o acercan a la plenitud en su condición de seres humanos."

A partir del marco anterior, son codificados los discursos de los inmigrantes venezolanos mediante conceptos subyacentes de las experiencias vividas en la ciudad de Cúcuta; en ese orden de ideas, se tiene que la categoría jerárquica de percepción de la calidad de vida, abarca las categorías de segundo nivel, es decir, integración económica y social asociadas con las categorías de tercer nivel conformadas por aspectos negativos que perciben los migrantes.

Como se observa en la gráfica 1, la red hermenéutica expone que las integraciones social y económica son las principales categorías que forman parte de la percepción de la calidad de vida de los inmigrantes venezolanos en la ciudad de Cúcuta; en ello es posible apreciar que la categoría integración económica posee una proximidad 
semántica con la variable jerárquica, a partir de allí, se construye el primer foco de proximidad, demostrando una priorización del sentido material de la vida ante una situación de sobrevivencia, las categorías de inestabilidad económica, desempleo, falta de oportunidades y el subsistir, obstaculizan la consecución satisfactoria de una buena calidad de vida material. Para la población objeto de estudio, la protección y la estabilidad emocional está supeditada al sentido de propiedad y de tenencia de bienes materiales que brindan la confianza de tener el futuro asegurado.

"Nosotros realmente necesitamos dinero ¿por qué? Porque muchos queremos hacer cualquier cosa y no tenemos nada para empezar". Participante número 8, grupo focal 4.

Continuando el análisis, la categoría de integración social con una escasa proximidad a la categoría jerárquica, se ubica en un segundo plano, dentro del cual se demuestran dos tipos de discriminación, una exclusión cívica explicada por las categorías de discriminación y prejuicio, las cuales generan incertidumbre en los inmigrantes y otra por parte de los entes administrativos reflejada en la desatención, que como lo expresa Manga (2018) en su columna La incontenible invasión de los venezolanos: "Esta crisis retrata una vez más, el insólito y frívolo manejo de las prioridades $y$ del tiempo de nuestras autoridades, en contemplativa etapa de diagnóstico, mientras avanza en el país en forma incontrolable, la mayor catástrofe humanitaria de todos los tiempos". Esta combinación de factores negativos como la incertidumbre y la discriminación según Subirats et al. (2004, p.19), "hacen que las condiciones materiales como psíquicas, les impiden desarrollarse como seres humanos".

"En ocasiones recibo las tablas sobre la cabeza, o en ocasiones el buen trato de un colombiano, porque así pasa". Participante número 8 , grupo focal 4 .

De esta manera, las categorías que conforman el segundo foco de proximidad eventualmente generan un estigma que desemboca en las dos categorías que hacen parte de la intercepción: la exclusión social y falta de oportunidades. Las particulares ubicaciones de estas categorías se dan como el engranaje que impide que se desarrollen los dos tipos de integración. Lo anterior se explica primero, porque la falta de oportunidades ante una llegada precaria, se traduce en situaciones de pobreza que conllevan a la marginación y, en segundo lugar, por el significado de la categoría, la cual hace referencia a la falta de regularidad en los documentos que les permitan tener seguridad social, empleo, acceso a educación y representatividad, de manera que tras no obtener esto, se hace más difícil aprovechar las escasas oportunidades que brinda la ciudad de Cúcuta. 
Gráfica 1. Percepción de la calidad de vida de los migrantes venezolanos en la ciudad de Cúcuta

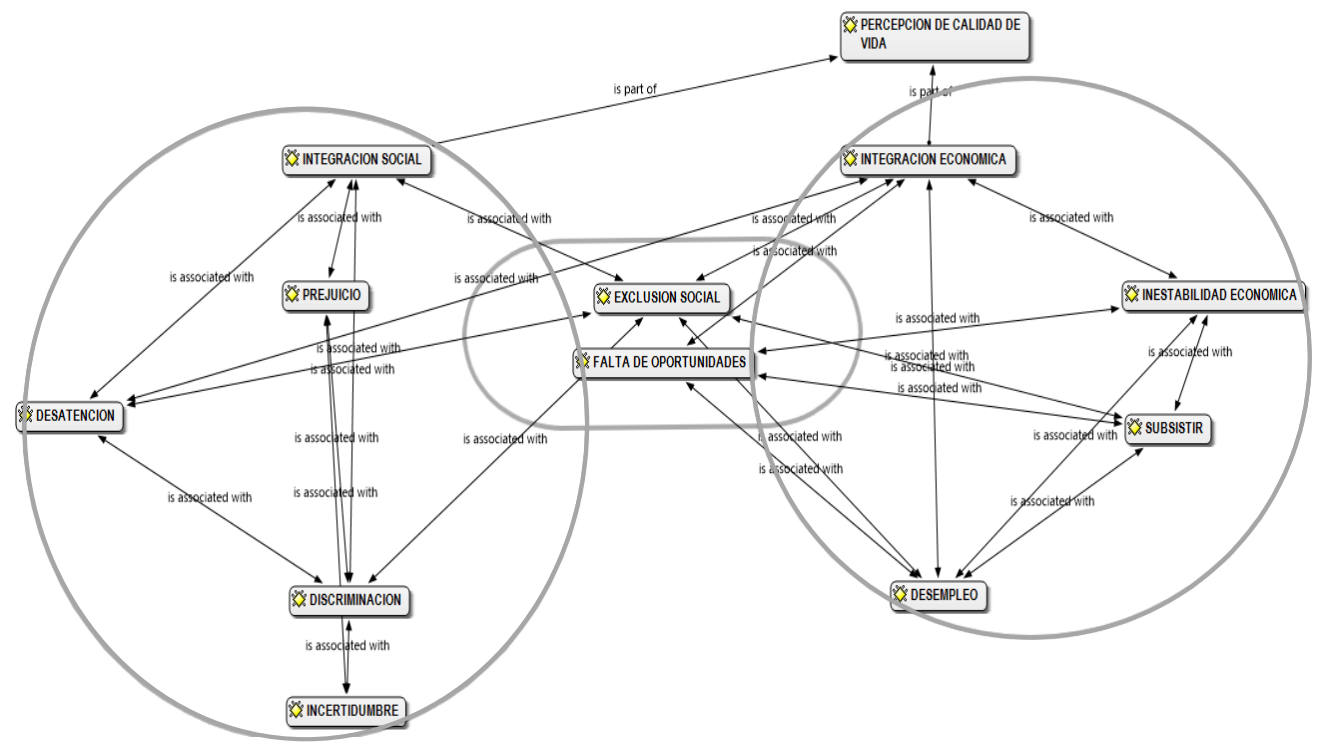

Fuente: elaboración propia

\subsubsection{Red semántica condiciones ideales}

En el imaginario de una buena calidad de vida de la población estudiada, se tiene que mediante las categorías propuestas por Max-Neef, más las categorías creadas a partir del discurso de los participantes, se configura una cosmovisión de desarrollo con la estructura explícita en el anexo 2.

Ahora bien, la red de condiciones ideales permite apreciar la proximidad de las categorías de necesidades axiológicas y existenciales con la categoría jerárquica, este es un primer foco de proximidad, donde es clara la priorización de las necesidades axiológicas frente a las existenciales. Seguidamente, un segundo foco de proximidad semántica, en donde las necesidades existenciales giran en torno a la categoría de estabilidad económica, siendo esta la más priorizada por los inmigrantes dentro de su visión de desarrollo.

Como se explicó párrafos atrás, esta visión material que ubica la economía como determinante fundamental del desarrollo, es propia de personas que migran buscando un mejor futuro. Así mismo, las categorías de hacer y tener se acercan más a la estabilidad económica, siendo estas las prioridades para suplir sus necesidades: tener un empleo, realizar actividades que generen ingresos y que les permitan su anhelada calidad de vida. 
Por otro lado, las dos categorías a los extremos son la protección y la subsistencia, posicionadas como elementos importantes del desarrollo, debido a su cercanía con las categorías de segundo orden, y que también están relacionadas con categorías de cuarto orden como el empleo, las oportunidades, la familia, el equilibrio emocional y la inclusión, estos son satisfactores sinérgicos y determinantes dentro de su perspectiva, ya que suplen varias necesidades al tiempo como la identidad, la protección, la subsistencia y el afecto. También se puede apreciar que esta visión de desarrollo es materialista, al relacionar la tenencia de bienes económicos con un mejor bienestar, pero no es coincidencia que la ausencia de estos es los que a lo largo del tiempo ha provocado los mayores fenómenos migratorios en el mundo.

Es de resaltar también un tercer foco que relaciona la estabilidad política como la generadora de oportunidades, su cercanía con la libertad y la democracia, expresan que dentro de la visión que incluye este sistema político como parte del desarrollo de los inmigrantes, se denotan características de la escuela neoliberal, la cual establece que el libre funcionamiento del mercado es el que asigna eficientemente los recursos y genera un aumento del bienestar económico, por lo tanto, un lugar desarrollado será aquel que brinde esas características; de igual manera, esta visión está influenciada por el sistema político del que provienen, que según los inmigrantes, es coartador de libertades en el ámbito económico y social.

Como se aprecia a lo largo del análisis de las redes, la visión del desarrollo en los inmigrantes venezolanos está determinada en mayor medida por la estabilidad económica, a pesar de ser un grupo afectado de manera psíquica por factores negativos a raíz de su nacionalidad, el interés económico prima por encima de su salud mental, así Jiménez y García (2013, p. 40) mencionan que:

La dinámica social aparece hoy tensionada por la homogeneización de la vida colectiva fomentada por la industrialización, la educación formalizada, las pautas de trabajo, la moda, el urbanismo, las tecnologías de la información, los medios de comunicación de masas, etc. todo ello ha contribuido a generar estilos de vida uniformes, derivados de la racionalización y modernización de costumbres y hábitos de origen occidental pero que ya son mundiales.

\section{CONCLUSIONES}

La integración de los inmigrantes venezolanos en la ciudad de Cúcuta se ha visto afectada en dos aspectos: social y económico. Desde la perspectiva de la población estudiada, las expectativas en el mejoramiento de su calidad de vida no se logran satisfacer, dado que señalan un fenómeno de discriminación explícita por parte de 
Gráfica 2. Red semántica del desarrollo de los migrantes venezolanos en la ciudad de Cúcuta

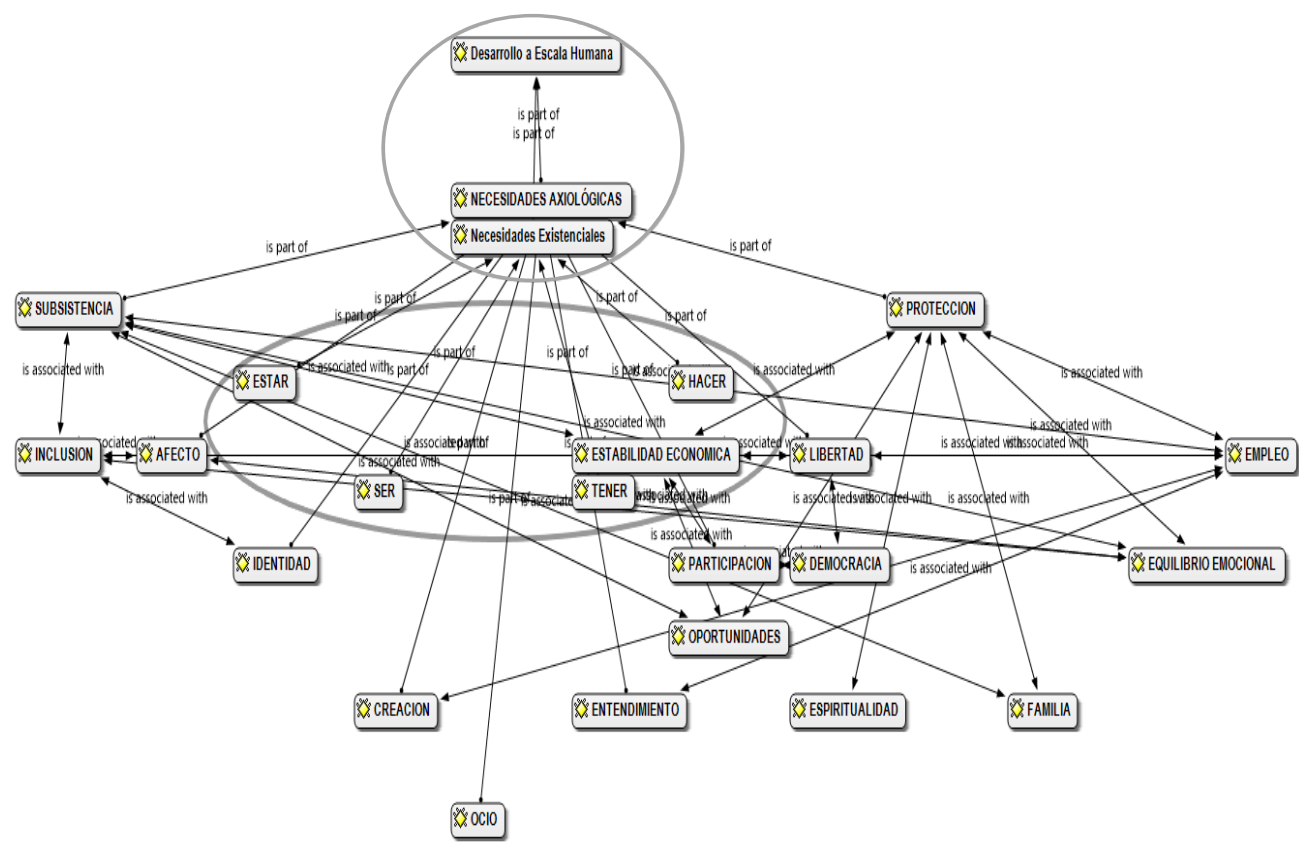

Fuente: elaboración propia.

la ciudadanía, e implícita, por parte de las administraciones locales, lo que conlleva a la exclusión al no ser beneficiarios de las políticas públicas locales. Eventualmente, la exclusión social y la estancia irregular afectan su participación en un mercado laboral que históricamente ha estado afectado por el desempleo y la informalidad, lo que se traduce en inestabilidad económica, elemento primordial de su escala de valoración.

Teniendo en cuenta que la ciudad brinda una alternativa frente al abastecimiento de productos básicos, supliendo de alguna forma necesidades como la subsistencia; la investigación refleja enormes diferencias en cada una de las necesidades axiológicas que comunican la situación actual e ideal de los inmigrantes venezolanos, lo cual expresa en primer lugar, que el desarrollo que encuentran en la ciudad no es el que esperarían y, en segundo lugar, que no hay un desarrollo a escala humana para la población inmigrante, ya que este consiste en la satisfacción de esas necesidades fundamentales, las cuales no se están supliendo de la manera correcta en la sociedad de acogida, y debido a lo anterior, el escenario más preocupante para ellos es el devolverse a su país. 
De alguna forma la visión de los inmigrantes se ha visto influenciada por las experiencias que forman parte su pasado, lo que conlleva a que una mejor calidad de vida esté relacionada con la estabilidad económica, política y el sentimiento de libertad, ejemplo de lo anterior se evidencia en el hecho de provenir de un país gobernado por un mismo partido político desde comienzos de siglo XXI, ubicando a la democracia como el mejor satisfactor de la libertad y la participación en un lugar desarrollado y con oportunidades. De igual manera, satisfactores de necesidades como la protección, incluyen, una casa, una moneda estable, actividades que les generen ingresos como un empleo y estar rodeado de vínculos sociales importantes como la familia, todo esto bajo las garantías de una estabilidad política que no se encuentra en la sociedad de expulsión.

\section{BIBLIOGRAFÍA}

Agudelo, Pedro. (2011). (Des) hilvanar el sentido/los juegos de Penélope. Una revisión del concepto imaginario y sus implicaciones sociales. En: Uni-Pluri/Versidad, vol. 11, n. ${ }^{\circ} 3$, p. 1-18.

Bañón, Alicia y Molina, María. (2013). Gestión de la diversidad: un reto actual ineludible. En: Sánchez-Molina, María Isabel. (Coord.), Los retos de la integración de los migrantes: una perspectiva multidisciplinar. Murcia: Laborum, p. 14-34.

Bustelo, Pablo. (1998). Teorías contemporáneas del desarrollo económico. Madrid: Síntesis, 304 p.

Cepal -Comisión Económica para América Latina y el Caribe-. (2018). Balance preliminar de las economías de América Latina y el Caribe 2017: República Bolivariana de Venezuela. Santiago de Chile: Cepal, 143 p.

DANE -Departamento Administrativo Nacional de Estadísticas-. (2011). Necesidades básicas insatisfechas (NBI). Bogotá: DANE.

DANE -Departamento Administrativo Nacional de Estadísticas-. (2018a). Pobreza monetaria y multidimensional en Colombia 2017. Bogotá: DANE, 45 p.

DANE -Departamento Administrativo Nacional de Estadísticas-. (2018b). Gran encuesta integrada de hogares (GEIH). Mercado laboral: indicadores de mercado laboral por ciudad trimestre octubre - diciembre 2017.

Girola, Lidia. (2008). Del desarrollo y la modernización a la modernidad. De la posmodernidad a la globalización. Notas para el estudio acerca de la construcción y el cambio conceptual, continuidades y rupturas en la sociología latinoamericana. En: Sociológica, vol. 23, n. 67 , p.13-32.

Hernández, Manuel y López, Diego. (2013). La situación residencial de los inmigrantes en España. En: Sánchez, María. Los retos de la integración de los migrantes: una perspectiva multidisciplinar. Murcia: Laborum, p. 169-197.

Hidalgo-Capitán, Antonio Luis. (2011). Economía política del desarrollo: la construcción retrospectiva de una especialidad académica. En: Revista de Economía Mundial, n. ${ }^{\circ}$ 28, p. 279-320. 
Hobsbawn, Eric. (1999), Historia del siglo XX. Buenos Aires: Crítica, 305 p.

Jiménez, Ana y García, José. (2013). Sociedades multiculturales: desafío a políticas integradoras. En: Sánchez, María. Los retos de la integración de los migrantes: una perspectiva multidisciplinar. Murcia: Laborum, p. 35-52.

Leal Buitrago, Francisco. (2012). Una mirada a las "ciencias militares" desde las ciencias sociales. En: Análisis Político, vol. 26, n. ${ }^{\circ} 78$, p. 211-219.

Manga, German. (2018). La incontenible invasión de los venezolanos. En: Semana, Bogotá, 1 p.

Max-Neef, Manfred; Elizalde, Antonio y Hopenhayn, Martín. (2010). Desarrollo a escala humana: opciones para el futuro. Barcelona: Icaria, $56 \mathrm{p}$.

Meier, Gerald. (2002). La vieja generación de economistas y la nueva. En: Meier, Gerald y Stiglitz, Joseph. (Eds.). Fronteras de la economía del desarrollo: el futuro en perspectiva. Ciudad de México: Alfaomega, p.1-38.

Migración Colombia. (2017). Radiografía migratoria Colombia. Bogotá: Migración Colombia, 8 p.

Migración Colombia (2018). Informe sobre la movilidad humana venezolana: realidades y perspectivas de quienes emigran. Bogotá: Migración Colombia, 26 p.

Mora Toscano, Oliver. (2006). Las teorías del desarrollo económico: algunos postulados y enseñanzas. En: Apuntes del Cenes, vol. 26, n. ${ }^{\circ} 42$, p. 49-74.

Ortiz, Pilar; Manzanera, Salvador y Clavero, Esther. (2013). La feminización de los procesos migratorios en España y la región de Murcia. En: Sánchez, María (Ed.), Los retos de la integración de los migrantes: una perspectiva multidisciplinar. Murcia: Laborum, p. 121-145.

Rosales, Osvaldo. (1988). Balance y renovación en el paradigma estructuralista del desarrollo latinoamericano. En: Revista Cepal, n. ${ }^{\circ}$ 34, p. 19-36.

Hernández Sampieri, Roberto; Fernández, Carlos y Baptista, Pilar (2014). Metodología de la investigación. Ciudad de México: McGraw-Hill, 634 p.

Subirats, Joan; Riba, Clara; Giménez, Laura; Obradors, Anna; Giménez, María; Queralt, Didac; Bottos, Patricio y Rapoport Ana. (2004). Pobreza y exclusión social: un análisis de la realidad española y europea. En: Colección de Estudios Sociales. Barcelona: Fundación la Caixa, vol. 16, $189 \mathrm{p}$.

Veiga de Cabo, Jorge; De la Fuente, Elena y Zimmermann, Marta. (2008). Modelos de estudio en investigación aplicada: conceptos y criterios para el diseño. En: Medicina y Seguridad del Trabajo, vol. 54, n. 210 , p. 81-88.

Zambrano, Mario; Araque, Leonardo y Ravelo, Diana. (2014). Movilidad laboral en la frontera Cúcuta y área metropolitana y los municipios de San Antonio del Táchira y Pedro María Ureña 2014. Pamplona: Universidad de Pamplona, 23 p. 


\section{ANEXOS}

\section{ANEXO 1. CLASIFICACIÓN DE CATEGORÍAS DE LA RED SEMÁNTICA NEGATIVA}

Categorías

\begin{tabular}{lcl}
\hline \multicolumn{2}{c}{ Categoría de jerarquía } & Categoría de asociación \\
\hline \multicolumn{1}{c}{ Primer nivel } & \multicolumn{1}{c}{ Segundo nivel } & Tercer nivel \\
\hline Percepción de la cali- & Integración social & Discriminación \\
dad de vida & Integración económica & Subsistir \\
& Incertidumbre \\
& Desatención \\
& Falta de oportunida- \\
& des \\
& Desempleo \\
& Exclusión social \\
& Inestabilidad econó- \\
& mica
\end{tabular}

ANEXO 2. CLASIFICACIÓN DE CATEGORÍAS DE LA RED SEMÁNTICA POSITIVA

Categorías

\begin{tabular}{llll}
\hline \multicolumn{2}{c}{ Categorías jerárquicas } & \multicolumn{2}{c}{ Categorías de asociación } \\
\hline \multicolumn{1}{c}{ Primer nivel } & \multicolumn{1}{c}{ Segundo nivel } & \multicolumn{1}{c}{ Tercer nivel } & \multicolumn{1}{c}{ Cuarto nivel } \\
\hline \multirow{2}{*}{$\begin{array}{c}\text { Desarrollo a Escala } \\
\text { Humana }\end{array}$} & $\begin{array}{l}\text { Necesidades existen } \\
\text { ciales }\end{array}$ & Ser & Familia \\
& Necesidades axioló- & Hacer & Espiritualidad \\
& gicas & Estar & Empleo \\
& Subsistencia & Oportunidades \\
& Protección & Estabilidad econó- \\
& Afecto & Equilibrio emocional \\
& Entendimiento & Inclusión \\
& Participación & Democracia \\
& Ocio & \\
& Creación & \\
& Identidad & \\
& Libertad &
\end{tabular}

Fuente: elaboración propia. 